\title{
Genetic Characterization of Binucleate Rhizoctonia Species Causing Web Blight on Azalea in Mississippi and Alabama
}

T. A. Rinehart and W. E. Copes, United States Department of Agriculture, Agricultural Research Service, Thad Cochran Southern Horticultural Laboratory, 810 Highway 26 West, Poplarville, MS 39470; and T. Toda and M. A. Cubeta, North Carolina State University, Department of Plant Pathology, Center for Integrated Fungal Research, 225 Partners III, Raleigh, NC 27695-7567

\begin{abstract}
Rinehart, T. A., Copes, W. E., Toda, T., and Cubeta, M. A. 2007. Genetic characterization of binucleate Rhizoctonia species causing web blight on azalea in Mississippi and Alabama. Plant Dis. 91:616-623.

Web blight on containerized azalea is an annual problem for commercial nurseries during summer months in the southern United States. Losses to web blight are associated with the cost of fungicide applications, delayed marketing of diseased plants, and plant death. Two hundred and eleven isolates of binucleate Rhizoctonia were recovered from azalea leaves with web blight symptoms from two nurseries in Mississippi and Alabama over 3 years. The internal transcribed spacer region (ITS) of the ribosomal DNA (rDNA) was sequenced from all isolates to determine genetic identity. A single anastomosis group (AG) of binucleate Rhizoctonia represented $92 \%$ of the samples collected from infected leaves. Genetic data and hyphal fusion experiments confirmed that these isolates belong to AG-U, which was recently identified from root and stem infections on miniature rose in Japan. Isolates of binucleate Rhizoctonia belonging to anastomosis groups AG-R, CAG-7 (=AG-S), and AG-G were also identified in the sample in low frequency. This is the first report of the occurrence of binucleate Rhizoctonia AG-U in the United States.
\end{abstract}

Additional keywords: Ceratobasidium, rhododendron

Fungi in the Rhizoctonia species complex are soilborne plant pathogens with a worldwide distribution (2). Rhizoctonia species cause web blight on some azalea cultivars (Rhododendron eriocarpum (Hayata) Nakai) from several azalea groups (Glen Dale, Kurume, Robin Hill, and Satsuki) during nursery production, especially under the hot, humid conditions in the southern and southeastern United States (22). Disease development in infested containers is believed to begin by growth of fungal mycelium across the pine bark potting medium surface, followed by colonization of mycelium into the crown of the plant. Symptoms of leaf infection include marginal and interveinal irregular necrotic lesions that progress to total leaf necrosis and leaf-drop (22). Fallen leaves often dangle by mycelium attached to stems, thus the common name "web blight". While moderate damage is usually not lethal, unsightly defoliating effects can

Corresponding author: T. A. Rinehart

E-mail: trinehart@ars.usda.gov

Accepted for publication 11 December 2006.

doi:10.1094/PDIS-91-5-0616

This article is in the public domain and not copyrightable. It may be freely reprinted with customary crediting of the source. The American Phytopathological Society, 2007. prompt rejection of infected plants by customers. Cultural and management practices are the most effective and practical means of minimizing loss during production of container-grown azaleas. However, with the current control practices, web blight annually causes 2 to $10 \%$ of susceptible cultivars to be unmarketable (W. Copes, personal communication).

The etiology of species of Rhizoctonia causing web blight in nursery production has not been extensively examined. Web blight was first reported on azalea in 1966 (22). Koch's postulates were demonstrated for binucleate Rhizoctonia anastomosis groups CAG-7 and $R$. solani anastomosis group AG-1, as causative agents of Rhizoctonia web blight of azalea $(4,5)$. After further study, Frisina and Benson (5) concluded that web blight on azaleas was predominately caused by species of binucleate Rhizoctonia, but they did not determine anastomosis groups (5). Damping-off and root rot diseases caused by Rhizoctonia spp. and $R$. solani are reported on Rhododendron spp., but no research has been reported for these diseases on azalea (3). An understanding of the genetic diversity and identity of Rhizoctonia species causing web blight is critical for developing disease management practices and better understanding of epidemiology.

Binucleate Rhizoctonia can be divided into 21 anastomosis groups (AG) based on hyphal fusion criteria $(10,12-14)$. Sequence data for the internal transcribed spacer (ITS) regions of the ribosomal DNA for representative isolates of each AG are available in GenBank $(7,21)$. In the absence of a priori knowledge of the Rhizoctonia fungi associated with web blight disease of azalea, DNA sequence information was used to rapidly characterize and identify fungi causing disease in this study. This approach is attractive given the expenditure of labor and subjectivity associated with hyphal anastomosis testing. Several studies on binucleate Rhizoctonia have utilized DNA sequence data in conjunction with traditional hyphal anastomosis experiments and morphological descriptions to characterize pathogens on strawberry, cut flowers, and miniature roses $(10,11,16)$. Here we adopted these techniques to rapidly characterize a large number of samples collected from diseased azaleas.

The objective of this study was to characterize and identify species of Rhizoctonia causing web blight on azalea during production in commercial nurseries in Alabama and Mississippi over a 3-year period. Our hypothesis was that similar and specific AGs of Rhizoctonia species are causing web blight disease on azalea at both nursery locations over time. Our experimental approach for characterization and identification involved sequencing of ITS-rDNA, followed by nuclear staining and hyphal anastomosis testing. Subsequent experiments were performed to examine pathogenicity and phenotypic characteristics of the isolated fungi Preliminary reports of this data have been previously published $(17,18)$.

\section{MATERIALS AND METHODS}

Sampling strategy. Leaves with marginal and/or interlaminar necrotic lesions, or completely necrotic leaves, were collected from one nursery in Lucedale, MS, and one nursery in Wilmer, AL, on 16 and 20 August, 13 September, and 16 October in 2002; 10 and $25 \mathrm{July}, 8,21$, and $28 \mathrm{Au}-$ gust, and 1 and 3 September in 2003; and 18,25 , and 30 June, 6, 7, 19, and 20 July, and 20 and 21 August in 2004. Both nurseries are located in USDA hardiness zone $8 \mathrm{a}$, and the nurseries are $24 \mathrm{~km}$ apart. All plants growing on container pads were surveyed for web blight disease on collec- 
tion dates, and samples were collected from arbitrarily selected plants showing web blight symptoms. To identify duplicate samples, or samples collected from infected leaf tissue from the same plant on different collection dates, barcode labels were attached to each container sampled so pots could be identified and recorded if the same plant was sampled at a later date. A sample consisted of one to seven leaves collected with sterile forceps from a single plant. In 2002 and 2003, samples were placed directly on water agar (WA) in a 100-mm-diameter plastic petri plate in the field. In 2004, samples were placed in sterile $15-\mathrm{ml}$ plastic centrifuge tubes, set in a cooler with ice packs, and plated that same day on WA. In addition to location and host cultivar, data for all 2004 samples included GPS location, pot size, pot placement on pad (edge, interior, adjacent to pathway), disease severity, spacing of host plants, and host cultivar.

Within 3 days of plating symptomatic leaf tissue, the leading edge of mycelium was transferred to potato dextrose agar (PDA). Additional transfers were made as needed to obtain pure cultures of each isolate. Within 3 to 5 days, a $0.5 \times 1.0 \mathrm{~cm}$ block of agar with a leading edge of mycelia was submersed in $2.5 \mathrm{ml}$ of $15 \%$ glycerol in a 3.6-ml cryogenic tube at $-76^{\circ} \mathrm{C}$ for long-term storage. A smaller number of samples was additionally stored by transferring a $0.8 \times 1.0 \mathrm{~cm}$ block of agar with a leading edge of mycelium into a sterile 250-ml Erlenmeyer flask containing sterile moist barley, allowed to grow at $21^{\circ} \mathrm{C}$ for 14 days, then stored at $-10^{\circ} \mathrm{C}$.

PCR amplification and ITS sequencing. A single infested barley grain was placed on a piece of $\mathrm{P} 2$ filter paper resting on top of PDA and grown for 7 days. Mycelium was removed by peeling the filter paper off the agar. Total genomic DNA was isolated using UltraClean Soil DNA Extraction Kit (MoBio Laboratories, Carlsbad, CA) and quantified with picogreen (Molecular Probes, Eugene, OR) using a Turner Quantech Fluorometer (Barnstead/Thermolyne, Dubuque, IA). ITS regions of rDNA were amplified using PCR primers ITS1F and ITS4B and cycling conditions described by Gardes and Bruns (6). MasterMix taq polymerase was used for all reactions according to manufacturer's recommendations (Eppendorf, Westbury, NY).

Amplified products were purified using Qiaquick PCR purification columns (Qiagen, Valencia, CA) and sequenced directly using Big Dye version 3.1 and ABI3100-Avant capillary array sequencer (Applied Biosystems, Foster City, CA). Sequence data were generated by primers Rh3 (5' CAAGGTGCGTTCAAAGA 3') and Rh4 (5' ACAAGGTTTCCGTAGGTG $3^{\prime}$ ), which sequenced a 386-bp fragment covering ITS1 region, and Rh31 (5' CCTTGGAGCATGCCTGTTTG $3^{\prime}$ ) and
Rh34 (5' CGCTTCACTCGCCGTTACTA $3^{\prime}$ ), which sequenced $377 \mathrm{bp}$ of the ITS2 region. These internal sequencing primers anneal to conserved rDNA regions (18s, $5.8 \mathrm{~s}$, and $28 \mathrm{~s}$ RNA) to produce short, overlapping fragments in order to speed automated DNA sequence generation and assembly. Forward and reverse sequences were generated for each ITS region, and aligned and edited with Sequencer software (GeneCodes, Ann Arbor, MI). Mixed bases, as identified by $50 \%$ peak height using the $\mathrm{KB}$ basecaller, were recorded using IUPAC codes. Fourteen isolates of binucleate Rhizoctonia representing nine different anastomosis groups with rDNA sequences previously deposited in GenBank were processed in parallel with field samples as positive controls for the ITS PCR protocol (GenBank accession nos. AF354092, AF354089, AB196650, AB122145, AF354094, DQ085501, AF354081, AY927320). The accuracy of PCR amplification and DNA sequencing in our laboratory was confirmed by comparing DNA sequences deposited in GenBank.

Sequence distance and phylogenetic analyses. Sequence data for ITS1 and ITS2 regions were aligned for 211 unknown isolates (GenBank accession nos. DQ858477-DQ858898), the 14 tester isolates described above as positive controls, and 51 sequences from GenBank representing 21 different AG of binucleate Rhizoctonia (GenBank accession nos. AB122144, AB122145, AB 196639, AB196640, AB196641, AB196642, AB 196643, AB 196644, AB196645, AB 196646, AB 196647, AB 196648, AB 196649, AB196650, AB196651, AB 196652, AB196653, AB196654, AB196655, AB196656, AB196657, AB 196658, AB196659, AB 196660, AB196661, AB196662, AB196663, AB196664, AB196665, AB196666, AB214366, AB214367, AF354080, AF354081, AF354082, AF354083, AF354084, AF354085, AF354086, AF354088, AF354089, AF354090, AF354091, AF354092, AF354093, AF354094, AF354095, AF354097, AJ419932, AJ427400, AJ427407). Sequences were aligned with MegAlign (DNAstar, Madison, WI) using Clustal W and then modified by visual inspection of the generated alignments. The combined alignment was 639 nucleotides and included both ITS1 and ITS2 regions but not the connecting $5.8 \mathrm{~S}$ region. Genetic relatedness of isolates to each other and to reference samples was determined by maximum parsimony methods with PAUP* (Phylogenetic Analysis Using Parsimony, version 4.05b10) (20). Computational parameters included heuristic search using random sequence addition and treebisection-reconnection (TBR) as the branch-swapping algorithm. Gaps were manually introduced to increase similarity between sequences but treated as missing. The number of variable and phylogenetically informative sites was determined, and a strict consensus tree was computed. Bootstrap values from 100 replicates were calculated from a reduced dataset where identical data were only represented by a single DNA sequence.

Hyphal anastomosis testing and nuclear staining. Ten isolates, five from phylotype I and five from phylotype II (GenBank accession nos. DQ858479, DQ858482, DQ858493, DQ858497, DQ858595, DQ858498, DQ858561, DQ858573, DQ858592, and DQ858649), were tested for hyphal fusion to each other and AG-U reference sample MWR26 obtained from Mitsuro Hyakumachi in Japan. Isolates were grown on PDA from infested barley stored at $-10^{\circ} \mathrm{C}$. Nuclear staining and anastomosis pairing were conducted using the following procedure. Isolates were grown on PDA for 3 days at $25^{\circ} \mathrm{C}$ in the dark. Mycelial plugs ( $5 \mathrm{~mm}$ diameter) taken from the advancing margin of each isolate were paired $2 \mathrm{~cm}$ apart on a glass slide covered with $2 \%$ water agar, placed in a plastic box $\left(100 \times 200 \times 30 \mathrm{~mm}^{3}\right)$ on a sterile paper towel moistened with sterile distilled $\mathrm{H}_{2} \mathrm{O}$ and incubated at $25^{\circ} \mathrm{C}$ in the dark for 24 to $48 \mathrm{~h}$. Three replicates were prepared from each pairing combination. After 24 to $48 \mathrm{~h}$, the overlapping portion of mycelium was stained with one drop each of $0.6 \%$ phenosafranin (\#199648, SigmaAldrich, St. Louis, MO) and 3\% KOH. The nuclear condition of hyphal cells and somatic interactions were determined by observation at $\times 400$ magnification. Hyphal interactions were scored according to the scale developed by Carling (1), where C0 indicates no hyphal fusion, $\mathrm{C} 1$ involves hyphal contact with cell wall but no cytoplasmic fusion, $\mathrm{C} 2$ indicates cell wall and cytoplasmic fusion accompanied by pore formation and death of adjacent cells, and C3 indicates cell wall and cytoplasmic fusion with no death of fused cells. Pairings were performed in laboratories in Mississippi and North Carolina to confirm results.

Disease severity. A measure of pathogenicity and virulence was obtained by placing a single barley grain infested with a single isolate of Rhizoctonia fungi in the middle of the newest leaves at the end of a detached azalea 'Gumpo' stem approximately $6 \mathrm{~cm}$ long with $14.2 \pm 2.9$ (mean \pm standard error) leaves per stem. Stems were collected 4 to $18 \mathrm{~h}$ before inoculation. Stems collected $18 \mathrm{~h}$ prior were stored overnight in a resealable plastic bag with a damp paper towel at $5^{\circ} \mathrm{C}$. Within 4 $\mathrm{h}$ before inoculation, stems were rinsed in sterile deionized water (SDW) and disinfested in $0.06 \mathrm{~g}$ a.i. sodium hypochlorite liter $^{-1}$ (household bleach) for $60 \mathrm{~s}$. The cut end of a stem was placed in a 0.5 -dram glass vial (Fisherbrand, Atlanta, GA) that contained $0.3 \mathrm{ml}$ SDW. Parafilm was 
wrapped around the stem at the open end of the vial, and the stem/vial unit was placed in a 100 -mm-diameter plastic petri plate that contained $2 \mathrm{ml}$ of SDW to provide $>90 \%$ relative humidity. Petri plates were loaded into 15 stackable wooden upright but SDW from spilling from the plates. A noninoculated check (sterile barley grain) and inoculated check (isolate Rh291, chosen at random) was done with each day's selection of isolates. Isolates were inoculated onto three separate stems, each stem being a replication. A completely randomized design was used for plate placement in wooden racks at $21^{\circ} \mathrm{C}$.

Four days after being inoculated, every leaf of a stem was rated for disease severity (ordinal categorical scale of percent leaf area exhibiting necrosis: 0 [0\%], 0.25 [1 to 50\%], 0.75 [50 to 99\%], 1.00 [100\%]). Disease severity for a detached stem was calculated as: $\left[\left(0 \times n_{1}\right)+(0.25 \times\right.$ $\left.\left.n_{2}\right)+\left(0.75 \times n_{3}\right)+\left(1.00 \times n_{4}\right)\right] / n_{t}$, where $n_{1}, n_{2}, n_{3}$, and $n_{4}$ are the numbers of leaves rated with the categorical value of the multiplier and $n_{t}$ is the total number of leaves. Experiment one was conducted with 112 AG-U isolates including 52 collected from the Alabama nursery and 60 from the Mississippi nursery in 2004 which had been recovered from 11 azalea cultivars (GenBank accession nos. DQ858533, DQ858535-DQ858538, DQ858541DQ858558, DQ858560-DQ858567, DQ858578, DQ858583, DQ858585, DQ858588, DQ858589, DQ858591DQ858593, DQ858595, DQ858596, DQ858600, DQ858603, DQ858611, DQ858613, DQ858615, DQ858618, DQ858619, DQ858621-DQ858637, DQ858639-DQ858654, DQ858656DQ858659, and DQ858661-DQ858687). Ninety-six were determined to be AG-U phylotype I and 16 were AG-U phylotype II. Due to disruption in electrical service and additional facility problems that resulted from Hurricane Katrina on $29 \mathrm{Au}-$ gust 2006, the ultra-low freezer that contained the isolates was inoperable for 4 weeks, and the majority of our cultures were lost. However, a second experiment with 11 surviving isolates was conducted (GenBank accession nos. DQ858547, racks at a $70^{\circ}$ angle to keep stems mostly

DQ858552， DQ858553， DQ858592, DQ858593， DQ858595, DQ858649, DQ858656, DQ858666, DQ858669, DQ858686, and DQ858687). Seven isolates were AG-U phylotype I and 5 were phylotype II. In experiment two, disease symptoms were not evident 4 days after inoculation; therefore, leaf incidence and severity ratings were assessed 8 days after inoculation.

Phenotypic traits. Physical characters were recorded for the same 112 AG-U isolates used in the disease severity analysis. After 17 to 28 days of growth on PDA at $21^{\circ} \mathrm{C}$, two traits, growth habit on agar surface and the nature of the aerial mycelium, were recorded from the top surface of PDA. Growth habit of mycelium on the agar surface consisted of six nominal categories: 1, uniform growth; 2, concentric band of dense aggregated growth located toward the middle of the plate with multiple radial indented grooves at intervals across the aggregated growth; 3 , concentric band of dense aggregated growth located toward the outer part of the plate with multiple radial indented grooves across the aggregated growth; 4, densely aggregated growth covering much of the plate area with a rough surface texture to the aggregated growth; 5, densely aggregated growth located toward the middle of the plate with a rough surface texture to the aggregated growth; and 6, densely aggregated growth located toward the outer portion of the plate with a rough surface texture to the aggregated growth. Habit of aerial mycelium consisted of two nominal categories: floccose and tomentose, each with 3 subcategories of slight, moderate, and dense. Floccose was defined as irregular wooly growth with a clumped to tufted or matted appearance. Tomentose was defined as finely stranded to wooly mycelium that is regularly spaced and evenly dispersed or with a groomed appearance.

Two traits were recorded from the reverse side of the petri plate. Percent area that was $\tan$ consisted of five ordinal categories: 1 (1 to 20); 2 (21 to 40 ); 3 (31 to 60); 4 (61 to 80); 5 (81 to 99). Distribution pattern of the $\tan$ area consisted of five nominal categories: 1, tan color mostly concentrated in the center of the plate; 2 ,

Table 1. Number of isolates from binucleate Rhizoctonia anastomosis groups (AG) recovered from infected azalea cultivars in Alabama and Mississippi in 2002, 2003, and 2004

\begin{tabular}{llrrrr}
\hline & & \multicolumn{3}{c}{ Year } & \\
\cline { 3 - 5 } Location & Anastomosis group (AG) & $\mathbf{2 0 0 2}$ & $\mathbf{2 0 0 3}$ & $\mathbf{2 0 0 4}$ & Total \\
\hline Alabama & AG-U Phylotype I & 5 & 20 & 52 & 77 \\
& AG-U Phylotype II & 0 & 2 & 13 & 15 \\
& CAG7(=AG-S) & 1 & 5 & 4 & 10 \\
& AG-R & 1 & 0 & 0 & 1 \\
& AG-G & 0 & 0 & 0 & 0 \\
Mississippi & AG-U Phylotype I & 9 & 12 & 77 & 98 \\
& AG-U Phylotype II & 1 & 0 & 5 & 6 \\
& CAG7(=AG-S) & 0 & 0 & 1 & 1 \\
& AG-R & 0 & 0 & 2 & 2 \\
& AG-G & 0 & 0 & 1 & 1 \\
\hline
\end{tabular}

tan color mostly concentrated as a band(s) in the middle section of radial growth; 3 , tan color mostly concentrated as a band(s) in the outer section of radial growth; 4 , tan color regularly or irregularly patterned across the plate; 5, tan color uniformly dominate across the plate.

Statistical analysis. Disease severity was analyzed with an analysis of variance method of mixed models using Proc Mixed (version 9.1.3, SAS Institute, Cary, NC). Analysis of experiment one (112 isolates) was done on anastomosis group, nursery, and cultivar using random effects in the error term to account for variance due to replication and to nursery and replication on phylotype. Phenotypic traits were analyzed with univariate analysis methods using Proc Freq since the nonparametric method does not require data to be normally distributed (SAS Institute, Cary, NC). Traits per isolate were recorded from three replicate plates. Since replication is not appropriate in this analysis, the mode value of the trait level per isolate was assigned based on the judgment of the assessor. For the majority of isolates, the three replications had the same value.

\section{RESULTS}

ITS data generated from 211 isolates of Rhizoctonia recovered from plants with web blight over 3 years included 17, 39, and 155 isolates in 2002, 2003, and 2004, respectively (Table 1 ). The samples for each year represent isolates collected from plants in separate pots. Spatial analysis was not appropriate because sampling involved an arbitrary selection of typically five to seven symptomatic plants within a block of plants grouped by the same cultivar and age within the nursery, without regard to the total number of plants and symptomatic plants within the block.

Four anastomosis groups of binucleate Rhizoctonia were identified in this study (Table 1). The predominant anastomosis group, AG-U, represented $92 \%$ of the sample. Samples characterized as AG-U were divided into two distinct phylotypes based on sequence analysis of the ITS rDNA region. For our purposes, "phylotype" was defined as a group of PCR products that have pairwise similarity of $99 \%$ or greater. AG-U phylotype I represented 175 isolates, while phylotype II only contained 21 isolates. These isolates were recovered from 11 different azalea cultivars (Table 2). Both phylotypes of AG-U were represented in the sample from both nurseries, but phylotypes were unevenly recovered from cultivars. Cultivars were not present in equal number at both locations. Sixty percent of the AG-U isolates were recovered from two ('Gumpo Pink' and 'Gumpo White') of 12 cultivars with web blight (Table 2). Fifteen isolates representing three additional AG (CAG-7 [=AG-S], AG-R, and AG-G) were also identified in the sample (Table 1). 
Hyphal AG predictions were based on genetic relatedness to reference samples as determined by maximum parsimony methods (Fig. 1). Sixty-two variable sites were not informative, so parsimony analysis was based on 279 parsimony-informative characters. One predominant AG of binucleate Rhizoctonia, AG-U, appears to have consistently caused the majority of damage to nursery grown azaleas over 3 years. ITS sequence data from these isolates closely resembled DNA sequence data for reference isolate MWR26, a binucleate Rhizoctonia from miniature rose in Japan that belongs to AG-U $(10,16)$. ITS sequence data from the five isolates of AG-U from Japan were $100 \%$ identical to each other and $100 \%$ identical to 157 of the 175 samples in AG-U phylotype I. While maximum parsimony suggested phylotype II is related to AG-U, ITS sequence data for phylotype II was only $92 \%$ similar to phylotype I and the AG-U data from Japan (Fig. 2).

AG predictions were confirmed with hyphal fusion experiments. Isolates identified as belonging to a specific AG exhibited a $\mathrm{C} 2$ reaction when paired with the tester isolate for that specific AG and a C3 reaction when paired with itself. Despite the separation into two phylotypes based on the sequence data, hyphal fusion experiments between AG-U phylotype I and AG-U phylotype II revealed a $\mathrm{C} 2$ reaction when isolates within and between phylotypes of AG-U were paired. However, in the pairing of isolates within phylotype II, cell death was confined to the interacting cells and not observed beyond the interacting cells.

Mean disease severity ratings for the 112 isolates of experiment one were not significantly different due to phylotype ( $P$ $=0.0644)$, cultivar $(P=0.2461)$, or nursery $(P=0.6234)$. For the 112 isolates, disease severity values were $0.373 \pm 0.021$ (mean \pm standard error) and $0.304 \pm 0.038$ for AG-U phylotype I and II, respectively.
Mean disease severity ratings, for the subset of 11 isolates from experiment one that were repeated in experiment two, were not significantly different due to experiment $(P$ $=0.3218)$ or phylotype $(P=0.5333)$. For the 11 isolates, disease severity values were $0.342 \pm 0.048$ and $0.379 \pm 0.057$ for AG-U phylotype I and II, respectively. However, disease symptoms were measured at 4 and 8 days after inoculation for experiment one and two, respectively. A single isolate, Rh00209 (GenBank accession no. DQ858592), had a disease severity rating of 0.44 and 0.00 in experiments one and two, respectively, and was dropped from the analysis.

Both phylotypes of AG-U included isolates with variable phenotypic traits in culture. Phylotypes differed in the proportional number of isolates with the habit of mycelium on the agar surface, the habit of aerial mycelium, and the percent tan area, but were not different in the distribution of tan and dark areas (Table 3). Ninety-five percent of the AG-U phylotype I isolates had concentric dense areas of aggregated mycelium growth that were either roughly textured on the surface or with radially oriented indented grooves. While 59\% of the AG-U phylotype II isolates had those traits, $41 \%$ of the isolates had a flat mycelium growth that was not densely aggregated. The habit of aerial mycelium was also significantly different between isolates of AG-U phylotype I and II. Phylotype I isolates of AG-U were mostly tomentose $(89 \%)$, while isolates of AG-U (II) were typically floccose $(69 \%)$. Most isolates had two contrasting colors (shades of tan including copper or bronze and shades of brown to very dark brown) that occurred in 1 to 3 sets of alternating tan and brown concentric bands on the reverse side of the plate. AG-U phylotype I had a higher percentage of isolates with $>80 \%$ tan area, and AG-U phylotype II had a higher percentage of isolates with $<41 \%$ $\tan$ area.

\section{DISCUSSION}

This is the first confirmation of AG-U isolates of binucleate Rhizoctonia causing web blight on azaleas in the United States. The recovery of AG-U from infected azaleas parallels research by Priyatmojo et al. (16), who identified AG-U as causing root and stem rot on cut flowers and miniature roses. However, the frequency of isolation of AG-U in the studies of Priyatmojo et al. differed from our results. Of the 153 isolates obtained from infected roots and stems of miniature roses, only nine $(5.9 \%)$ were identified as belonging to AG-U (16). In our study of web blight on azaleas, AG$\mathrm{U}$ was recovered from more than $92 \%$ of the infected plants.

While all plants in each nursery (more than 45 azalea cultivars and 30 plant genera) were scouted for web blight at every sampling date in 2004 on both nursery sites, disease symptoms were found on only 12 azalea cultivars. In addition, disease symptoms were observed on more azalea cultivars at the Alabama nursery than at the Mississippi nursery, even though both nurseries grow most of the same azalea cultivars. Disease symptoms were not observed on any plant genera other than Rhododendron spp., although many genera can get Rhizoctonia web blight, such as: Abelia, Buxus, Cotoneaster, Elaeagnus, Gardenia, Hydrangea, Ilex, Juniperus, Lagerstroemia, Ligustrum, Pittosporum, and Raphiolepis.

In this study, only one isolate per plant was examined. Therefore, we were not able to determine whether both phylotypes of $\mathrm{AG}-\mathrm{U}$ or multiple anastomosis groups of binucleate Rhizoctonia occurred on a single infected plant. While fungi causing visible disease symptoms on azaleas were intensely sampled in this study, we did not randomly sample other azalea cultivars or plant genera without disease to establish the occurrence of AG-U on nonsymptomatic plants, the occurrence of nonpathogenic isolates of Rhizoctonia, or the overall

Table 2. Number of binucleate Rhizoctonia anastomosis groups (AG) recovered from azalea cultivars exhibiting web blight at one nursery in Alabama and one nursery in Mississippi

\begin{tabular}{|c|c|c|c|c|c|c|c|c|c|c|}
\hline \multirow[b]{2}{*}{ Azalea cultivar } & \multicolumn{5}{|c|}{ Alabama } & \multicolumn{5}{|c|}{ Mississippi } \\
\hline & $\begin{array}{l}\text { AG-U } \\
\text { Phylo- } \\
\text { type I }\end{array}$ & $\begin{array}{l}\text { AG-U } \\
\text { Phylo- } \\
\text { type II }\end{array}$ & $\begin{array}{c}\text { CAG7 } \\
\text { (=AG-S) }\end{array}$ & AG-R & AG-G & $\begin{array}{l}\text { AG-U } \\
\text { Phylo- } \\
\text { type I }\end{array}$ & $\begin{array}{l}\text { AG-U } \\
\text { Phylo- } \\
\text { type II }\end{array}$ & $\begin{array}{c}\text { CAG7 } \\
\text { (=AG-S) }\end{array}$ & AG-R & AG-G \\
\hline Azalea Red & 1 & - & - & - & - & - & - & - & - & - \\
\hline Christmas Cheer & - & - & - & - & - & 5 & 3 & - & - & - \\
\hline Conversation Piece & 9 & 2 & 1 & - & - & - & - & - & - & - \\
\hline Delaware Valley White & 1 & 1 & - & - & - & - & - & - & - & - \\
\hline Gumpo $^{z}$ & 13 & - & 4 & 1 & - & 12 & 1 & - & - & - \\
\hline Gumpo Pink & 18 & 6 & - & - & - & 31 & 1 & 1 & - & - \\
\hline Gumpo White & 22 & 2 & 3 & - & - & 26 & 1 & - & 2 & 1 \\
\hline Gyokushion & - & - & - & - & - & 5 & - & - & - & - \\
\hline Hershey Red & 3 & - & 2 & - & - & - & - & - & - & - \\
\hline Macrantha Pink & 4 & - & - & - & - & - & - & - & - & - \\
\hline Wakibisu & 3 & 3 & - & - & - & - & - & - & - & - \\
\hline Watchet & 3 & 1 & - & - & - & 19 & - & - & - & - \\
\hline Total & 77 & 15 & 10 & 1 & - & 98 & 6 & 1 & 2 & 1 \\
\hline
\end{tabular}

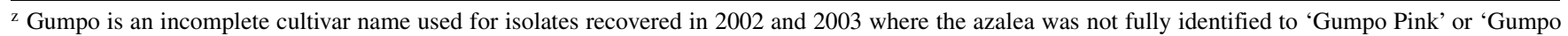
White'. 
distribution pattern of AG-U. Furthermore, the susceptibility of all azalea cultivars and plant genera to AG-U phylotypes at the two nursery sites is not known. Given the ubiquitous nature of Rhizoctonia fungi, it is prudent to assume that other populations are present on plant and soil in the nursery but not causing web blight.
While disease severity values were not significantly different between experiments, disease development required 4 days longer in experiment two than in

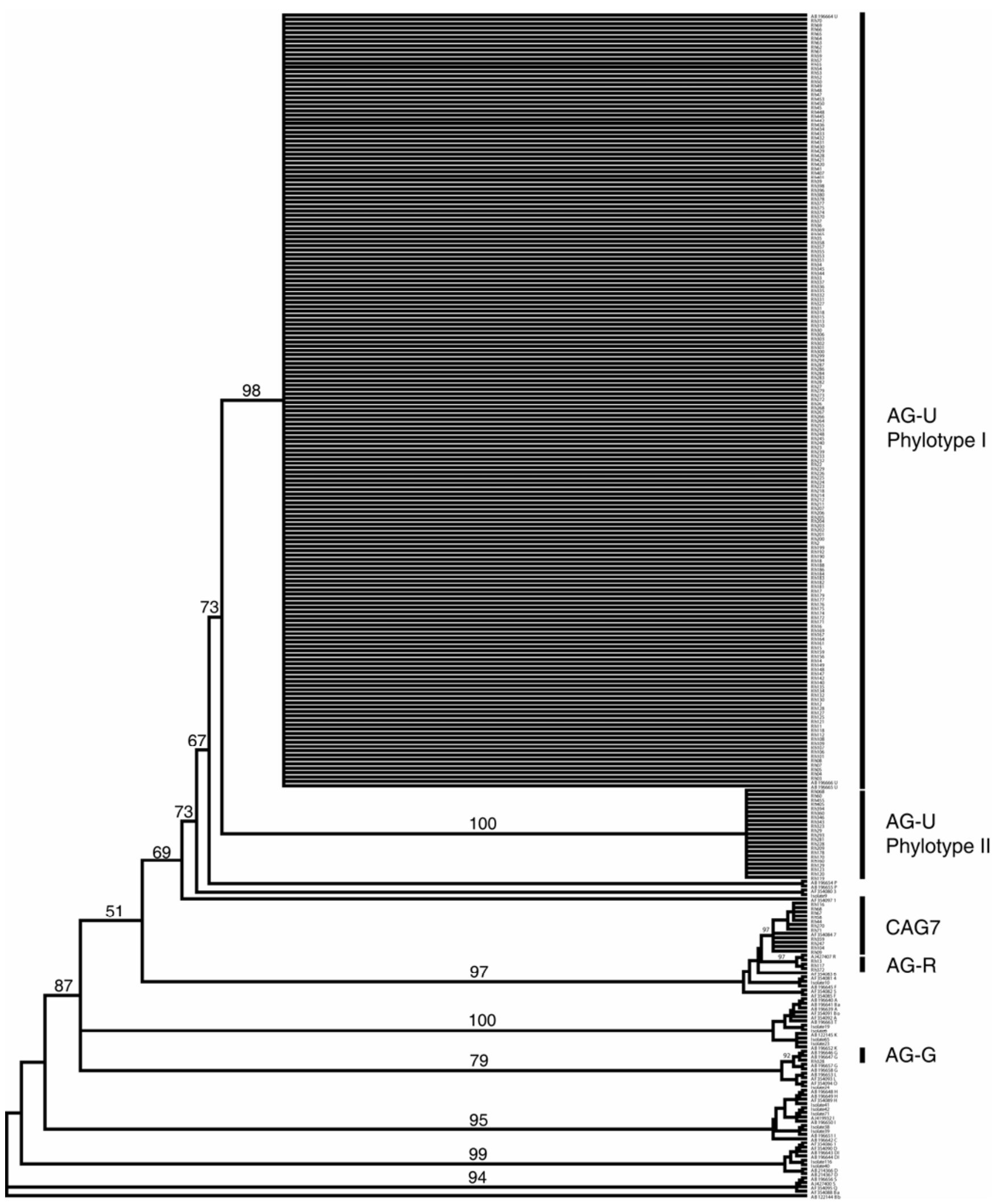

Fig. 1. A strict consensus tree was generated from the most parsimonious trees created from the combined alignment of internal transcribed spacer (ITS) 1 and ITS2 sequences. Total number of characters was 639 nucleotides with 341 variable sites and 279 parsimony-informative sites. Reference sequences are labeled by GenBank accession number followed by anastomosis group designation. Known isolates that were processed concurrent with field samples are included in the analysis to verify accurate generation of DNA sequence data. All other samples are labeled with an 'Rh' prefix and represent experimental samples collected from nurseries in Alabama and Mississippi over 3 years. 
experiment one. One possible explanation is that culture storage conditions may have affected pathogenicity, especially since experiments were repeated using isolates stored at $-78^{\circ} \mathrm{C}$ that experienced a 4-week period at ambient conditions during September in southern Mississippi after the passage of Hurricane Katrina and subsequent loss of electricity. A second possible explanation is that the initial experiment was done in September to early October when plant tissue was still acclimated to warm temperatures and the second experiment was done in January when plant tissue was hardened due to winter conditions. Interestingly, increased frequency of binucleate Rhizoctonia AG-U attacking miniature rose in Japan was not necessarily correlated with increased pathogenicity $(10,16)$, suggesting that the prominence of AG-U on azalea may be due in some degree to other ecological factors.
The frequency of phenotypic traits differed between phylotypes of AG-U. However, the phenotypic traits were variable for both phylotypes, and no category of a trait was uniquely characteristic to a specific phylotype; therefore, phenotypic traits would not be useful for preliminary identification of AG-U or its phylotypes. While our morphological characterization of AG$\mathrm{U}$ isolates was more detailed, the descriptions were comparable to those by Hya-

\title{
Internal Transcribed Spacer region 1 \\ ...GGCA--CTTGTGCACACCT-TCTCTTTCATCCACACACACCTGTGCACTT \\ ...GGCAATTTTGTGCACGCCTㅡTCTCTTTCATCCACACACACCTGTGCACTT
}

GTGAGACAGACTGTGGGGACTTTATTGAACCCCGTCTGTCTACTTAATTT GTGAGACAGA_TGTGGGGACTTTATTGAACCCCGTCTGTCTACTTAATCC

ACACACACTCTATTTAATTTAAACTGAATGTAATTGATGTAACGCATCTA ACACACACTCTATTTAATTTGAACTGAATGTAATTGATGTAACGCATCTA

\begin{tabular}{|c|c|c|c|c|}
\hline & \\
\hline & $\begin{array}{l}\text { ACTAAGTTTCAACAACGGATCTCTTGGCTCTCGCATCGATG. } \\
\text { ACTAAGTTTCAACAACGGATCTCTTGGCTCTCGCATCGATG. } \\
5.8 \mathrm{~s}\end{array}$ \\
\hline
\end{tabular}

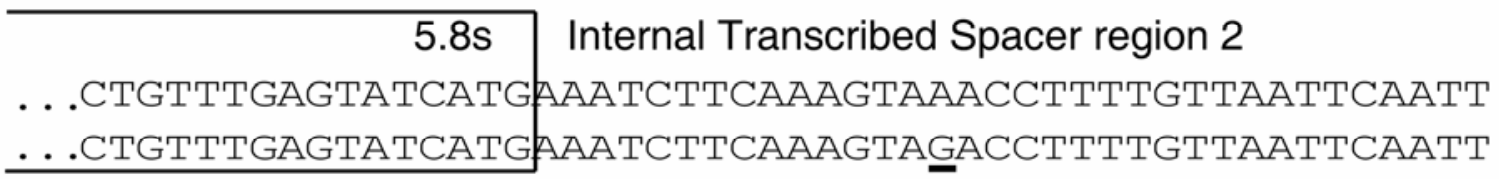

GGTTCCTTACTTTGGTATTGGAGGTCTTTGCAGTTTCACAACTGCTCCTC

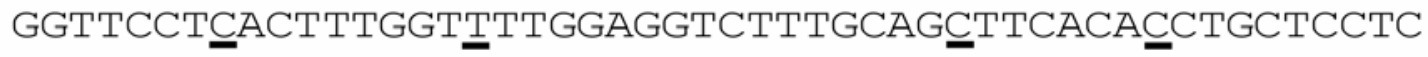
TTTGTTCATTAGCTGGATCTCAGTGTTATGCTTGGTTCCACTCGGCGTGA TTTGTTCATTAGCTGGATCTCAGTGTTATGCTTGGTTCCACTCGGCGTGA

TAAGTATCTATCGCTGAGGACACTTGTAAAAAGGTGGCCAAGGTAAACGC TAAGTATCTATCGCTGAGGACACT=GTAAAAAGGTGGCCAAGGTAAATGC

AGATGAACCGCTTCTAACAGTCCATTGACTTGGACAATA---AATATTTA AGATGAACCGCTTCTAACAGTCCATTGACTTGGACAAAATAAAATATTTA

TGATCTGATCTCAAATCAGGTAGGACTACCCGCTGAACTTAAGCATATCA TGATCTGATCTCAAATCAGGTAGGACTACCCGCTGAACTTAAGCATATCA

\author{
ATAAGCGGAGGAAAAGAAACTAACAAGGATTCCCCTAGTAACG. . . \\ ATAAGCGGAGGAAAAGAAACTAACAAGGATTCCCCTAGTAACG. . .
}

Fig. 2. Consensus sequences for anastomosis group (AG)-U phylotypes I and II. Data for phylotype I are shown above phylotype II and differences between the two are underlined. Boxed region is the conserved 5.8s rDNA between the internal transcribed spacer regions (ITS), 
kumachi et al. (10) including the observation and formation of sclerotia.

We choose to use ITS-rDNA sequence data to characterize fungal populations causing web blight on azaleas because it has been effective for characterizing fungal populations even without culturing or characterizing individual samples. For example, ITS-rDNA sequence data were used to assess diversity and uncover previously unknown fungal lineages in tundra soils (19). In our analyses we used the term phylotype to describe groups of isolates containing nearly identical or identical ITS-rDNA sequence data. Phylotype groups are generally used to describe microbial diversity using $16 \mathrm{~s}$ ribosomal DNA libraries made from communities that are difficult to sample but contain organisms that are not easy to culture in laboratory environments (15). We adopted this nomenclature to rapidly assess diversity in a large collection of isolates where prior knowledge is limited. Phylotype groups were based solely on $99 \%$ sequence similarity, and specific relationships or traditional taxonomic classification is not implied.

Molecular systematics has substantially advanced the taxonomy of Rhizoctonia species and other filamentous fungi (79,21). Comparing ITS-rDNA sequence data from unknown samples to data from reference samples can be a reliable indication of AG. For example, analysis of ITSrDNA sequence data was used in part to identify the binucleate Rhizoctonia species causing disease on strawberry (11). Here we used reference data from 21 binucleate
Rhizoctonia AG and maximum parsimony methods to predict AG status of hundreds of isolates collected from azaleas over 3 years. We had no prior knowledge of the Rhizoctonia species involved except that it was probably binucleate $(4,5)$. AG predictions were confirmed with traditional hyphal fusion experiments, which are still a useful and accurate means to identify binucleate Rhizoctonia.

Our results indicated AG-U may be the predominant causative agent of azalea web blight, which is relevant since it was relatively recently identified in Japan and isolates were not yet available from Rhizoctonia species collections in the United States $(10,16)$. In the absence of any published reports on the specific AGs of Rhizoctonia fungi associated with azalea, molecular data were a rapid and effective method to predict $\mathrm{AG}$ status and reduce the labor involved in gathering potential tester isolates for confirmation with anastomosis and nuclear staining. Given the increasing number of reference sequences available for comparison, our results suggest that ITS data can be used to characterize large numbers of unknown isolates and predict AG status for binucleate Rhizoctonia species infecting ornamental crops.

AG-U isolates of phylotype I from azalea in Alabama and Mississippi are closely related to the AG-U isolates from miniature rose in Japan. However, a second genetically related group with a different ITS-rDNA sequence (phylotype II) was recovered in low frequency from infected azalea leaves in Alabama and Mississippi. Hyphal anastomosis experiments con-

Table 3. Distribution of phenotypic trait characters between phylotypes (Pt) I and II of binucleate Rhizoctonia anastomosis group (AG)-U

\begin{tabular}{|c|c|c|}
\hline \multirow[b]{2}{*}{ Phenotypic trait } & \multicolumn{2}{|c|}{ Frequency $(\%)^{\mathrm{x}}$} \\
\hline & Pt I & Pt II \\
\hline \multicolumn{3}{|l|}{ Habit of mycelium on agar surface } \\
\hline Flat & 4.9 & 41.2 \\
\hline Concentric band of densely aggregated mycelium with radial grooves & 69.6 & 41.2 \\
\hline \multirow[t]{2}{*}{ Concentric band of densely aggregated mycelium with rough surface } & 25.5 & 17.6 \\
\hline & \multicolumn{2}{|c|}{$P=0.0004^{\mathrm{y}}$} \\
\hline \multicolumn{3}{|l|}{ Habit of aerial mycelium } \\
\hline Floccose (irregularly spaced mycelium that clump together in areas) & 10.8 & 68.8 \\
\hline \multirow[t]{2}{*}{ Tomentose (evenly and neatly spaced strands of mycelium) } & 89.2 & 31.2 \\
\hline & \multicolumn{2}{|c|}{$P<0.0001^{\mathrm{y}}$} \\
\hline \multicolumn{3}{|l|}{ Percent tan $\operatorname{area}^{\mathrm{z}}$} \\
\hline 5 to $40 \%$ & 9.8 & 41.2 \\
\hline 41 to $60 \%$ & 44.1 & 29.4 \\
\hline 61 to $80 \%$ & 24.5 & 23.5 \\
\hline \multirow{2}{*}{81 to $95 \%$} & 21.6 & 5.9 \\
\hline & \multicolumn{2}{|c|}{$P=0.0115^{\mathrm{y}}$} \\
\hline \multicolumn{3}{|l|}{ Distribution of $\tan$ area } \\
\hline Concentric band positioned centrally in plate & 43.2 & 17.6 \\
\hline Concentric band positioned intermediately in plate & 27.4 & 35.3 \\
\hline Concentric band positioned at outer edge of plate & 2.0 & 11.8 \\
\hline \multirow{2}{*}{ Regular throughout the plate } & 27.4 & 35.3 \\
\hline & \multicolumn{2}{|c|}{$P=0.0571^{\mathrm{y}}$} \\
\hline
\end{tabular}

$\overline{\mathrm{x}} n=96$ and 16 isolates for phylotypes I and II, respectively, were grown on potato dextrose agar at $21^{\circ} \mathrm{C}$ under a diurnal light-dark cycle for 17 to 28 days.

${ }^{y} P$ value for comparisons of the distribution of phylotype I and II isolates over categories within a phenotypic trait based on Fisher's exact test using the PROC FREQ procedure in SAS.

${ }^{\mathrm{z}}$ Percent tan area assessed as a nominal category on the reverse side of a culture plate. Shades of brown to dark brown accounted for the remainder of area. firmed that isolates from both phylotypes belong to AG-U and suggested that genetically distinct individuals may be present within each phylotype. However, additional research with multiple genetic markers is needed to determine whether isolates with the same ITS-rDNA sequence and differences in somatic compatibility alleles represent different genotypes. Additional genetic markers from other regions of the genome would be useful in comparing isolates of binucleate Rhizoctonia AG$\mathrm{U}$ from Japan (9).

While AG-U was the predominate cause of web blight, $8 \%$ of isolates causing azalea web blight were binucleate Rhizoctonia belonging to anastomosis groups AG-R, CAG7 (=AG-S), and AG-G. This information, along with recovery of multinucleate Rhizoctonia spp. from azaleas and the pine bark substrate of container grown azaleas, indicates that several Rhizoctonia spp. exist in the nursery (5; W. Copes, personal communication). The ecological and pathological significance of the diversity is unknown, especially since web blight symptoms were not found on other plant genera known to develop Rhizoctonia web blight.

Knowing that AG-U is a major pathogen causing web blight on azalea, at least at the two nurseries we sampled in Alabama and Mississippi, will help in the design of temporal and spatial studies since dispersal patterns should be affected by pathogen ecology. We can also begin testing the susceptibility of specific cultivars. The ultimate goal is to further develop fungicide timing and cultural disease control measures used in ornamental plant production, which differs dramatically from food and fiber crop production.

\section{ACKNOWLEDGMENTS}

Special thanks to Lavonne Stringer for isolate handling, Yonmee Han for DNA sequencing, and Debbie Boykin, USDA Midsouth Area Statistician, for statistical consultation. Mention of trade names or commercial products in this article is solely for the purpose of providing specific information and does not imply recommendation or endorsement by the U.S. Department of Agriculture.

\section{LITERATURE CITED}

1. Carling, D. E. 1996. Genetics of Rhizoctonia species. Pages 37-47 in: Rhizoctonia Species Taxonomy, Molecular Biology, Ecology, Pathology and Disease Control. B. Sneh, S. Jabaji-Hare, S. Neate, and G. Dijst, eds. Kluwer Academic, Dordrecht, Germany.

2. Cubeta, M. A., and Vilgalys, R. 2000. Rhizoctonia. Pages 109-116 in: Encyclopedia of Microbiology. Vol 4. J. Lederberg, ed. Academic Press, San Diego, CA.

3. Farr, D. B., Bills, G. F., Chamuris, G. P., and Rossman A. Y. 1995. Fungi on Plants and Plant Products in the United States. American Phytopathological Society, St. Paul, MN.

4. Frisina, T. A., and Benson, D. M. 1987. Characterization and pathogenicity of binucleate Rhizoctonia spp. from azaleas and other woody ornamental plants with web blight. Plant Dis. 71:977-981.

5. Frisina, T. A., and Benson, D. M. 1989. Occurrence of binucleate Rhizoctonia spp. on azalea 
and spatial analysis of web blight in containergrown nursery stock. Plant Dis. 73:249-254.

6. Gardes, M., and Bruns, T. D. 1993. ITS primers with enhanced specificity for basidiomycetes-Application to the identification of mycorrhizae and rusts. Mol. Ecol. 2:113-118.

7. Gonzalez, D., Carling, D. E., Kuninaga, S., Vilgalys, R., and Cubeta, M. A. 2001. Ribosomal DNA systematics of Ceratobasidium and Thanatephorus with rhizoctonia anamorphs. Mycologia 93:1138-1150.

8. González García, V., Portal Onco, M. A., and Rubio Susan, V. 2006. Review: Biology and Systematics of the form genus Rhizoctonia. Span. J. Agric. Res. 4:55-79.

9. Gonzalez-Hernandez, D., Vilgalys, R., and Cubeta, M. A. Phylogenetic utility of indels within sequences of ribosomal and betatubulin genes in fungi belonging to the Rhizoctonia species complex. Mol. Phylogenet. Evol. In press.

10. Hyakumachi, M., Kubota, M., Priyatmojo, A., and Fukui, H. 2005. New anastomosis groups, AG-T and AG-U, of binucleate Rhizoctonia spp. causing root and stem rot of cut-flower and miniature roses. Phytopathology 95:784-792.
11. Martin, F. N. 2000. Rhizoctonia spp. recovered from strawberry roots in central coastal California. Phytopathology 90:345-353.

12. Ogoshi, A., Oniki, M., Araki, T., and Ui, T. 1983. Anastomosis groups of binucleate Rhizoctonia in Japan and North America and their perfect states. Trans. Mycol. Soc. Jpn. 24:79-87.

13. Ogoshi, A., Oniki, M., Araki, T., and Ui, T. 1983. Studies on the anastomosis groups of binucleate Rhizoctonia and their perfect states. J. Fac. Agric. Hokkaido Univ. 61:244-260.

14. Ogoshi, A., Oniki, M., Sakai, R., and Ui, T. 1979. Anastomosis grouping among isolates of binucleate Rhizoctonia. Trans. Mycol. Soc. Jpn. 20:33-39.

15. Polz, M. F., and Cavanaugh, C. M. 1995. Dominance of one bacterial phylotype at a Mid-Atlantic ridge hydrothermal vent site. Proc. Natl. Acad. Sci. 92:7232-7236.

16. Priyatmojo, A., Yotani, Y., Hattori, K., Kageyama, K., and Hyakumachi, M. 2001. Characterization of Rhizoctonia spp. causing root and stem rot of miniature rose. Plant Dis. 85:1200-1205.

17. Rinehart, T. A., Copes, W. E., and Cubeta, M.
A. 2005. Genetic, pathogenic and phenotypic characterization of Rhizoctonia spp. isolated from container-grown azalea in the southern United States. (Abstr.) Phytopathology 95:S21.

18. Rinehart, T. A., Copes, W. E., and Cubeta, M. A. 2005. Genetic, pathogenic and phenotypic characterization of Rhizoctonia spp. isolated from container-grown azalea in the southern United States. Proc. Southern Nur. Assoc. Conf. 50:260-264.

19. Schadt, C. W., Martin, A. P., Lipson, D. A., and Schmidt, S. K. 2003. Seasonal dynamics of previously unknown fungal lineages in tundra soils. Science 301:1359-1361.

20. Swofford, D. L. 1998. PAUP*. Phylogenetic Analysis Using Parsimony (*and Other Methods). Version 4. Sinauer Associates, Sunderland, MA.

21. Toda, T., Hyakumachi, M., Kageyama, K., Suga, H., Tanaka, A., and Tani, T. 1999. Differentiation of Rhizoctonia AG-D isolates from turfgrass into subgroups I and II based on rDNA and RAPD analyses. Eur. J. Plant Pathol. 105:835-846.

22. Wehlbur, C., and Cox, R. S. 1966. Rhizoctonia leaf blight of azalea. Plant Dis. Rep. 50:354-355. 